\title{
Compression of remote sensing data using second-generation wavelets: a review
}

\begin{abstract}
Wavelet-based methods have been widely used for compression of remotely sensed images and data. Recently, second generation of wavelets which is based on a method called lifting has proven to be more effective than traditional wavelets as it provides lossless compression, lowers the memory usage, and is computationally faster. This study explores the literature related to applying second-generation wavelets for the compression of remote sensing data. Nevertheless, in order to compare the results of two wavelet types, some applications of traditional wavelets are also presented.
\end{abstract}

Keyword: Compression; Lifting scheme; Remote sensing; Second-generation wavelet 